\title{
Performance of a series of novel N-substituted acrylamides in capillary electrophoresis of DNA fragments
}

\author{
Ernesto Simò-Alfonso ${ }^{a}$, Cecilia Gelfi ${ }^{\mathrm{b}}$, Mara Lucisano ${ }^{\circ}$, Pier Giorgio Righetti ${ }^{\mathrm{d}, *}$ \\ "Universitat de Valencia, Departamento de Quimica Analitica. Facultad de Quimica, 46100 Burjassot, Spain \\ ${ }^{h} I T B A, C N R$, Via Ampere 56, Milano, Italy \\ 'Dip. Scienze e Tecnologie Alimentari e Microbiologiche. Universita' di Milano, Via Celoria 2, Milano 2013.3. Italy \\ "Department of Cell Biology. University of Calabria, Arcavacata di Rende. Cosenza. Italy \\ Received 16 April 1996; revised 12 July 1996; accepted 17 July 1996
}

\begin{abstract}
DNA separations by capillary electrophoresis in viscous solutions of novel polymers, made with $\Omega$-hydroxyl, $N$ substituted acrylamides (notably $\mathrm{N}$-acryloyl amino propanol, AAP and $\mathrm{N}$-acryloyl amino butanol, AAB) are evaluated. Whereas in standard poly(acrylamide), at $6 \%$ concentration, the theoretical plate number $(N)$ does not exceed 500000 , in $6 \%$ poly(AAP) $N$ reaches 922000 and in $6 \%$ poly(AAB) $N$ values as high as 1200000 are obtained. Also, copolymers of AAP and AAB give $N$ values in excess of 1 million plates. The two novel monomers (AAP and AAB) remain extremely stable during alkaline hydrolysis and display very good hydrophilicity, while being devoid of the noxious habit of autopolymerization and auto-reticulation exhibited by the previous monomer of this series ( $\mathrm{N}$-acryloyl amino ethoxy ethanol). The reasons for such a good performance of the $\Omega$-substituted acrylamide derivatives could be that their polymers may form hydrogen bonds via their distal $-\mathrm{OH}$ group during DNA separation.
\end{abstract}

Keywords: Acrylamides, N-substituted; N-acryloylaminopropanol; DNA

\section{Introduction}

Capillary zone electrophoresis (CZE) is a powerful technique for DNA analysis, which has found a number of applications in restriction fragment sizing, PCR product characterization, forensic identification and DNA sequencing (see Righetti and Gelfi for an extensive review [1]). Several recent developments have been described, such as capillary array electrophoresis [2-6] and CZE on chips [7-9]. Another

\footnotetext{
* Corresponding author. Address for correspondence: L.I.T.A., Via Fratelli Cervi 93, Segrate 20090, Milano, Italy.
}

area which has experienced marked progress is detection sensitivity. Recent reports, based on singlemolecule fluorescence burst detection, have demonstrated, in one case, a sensitivity, for large DNA fragments, of ca. 1000 DNA molecules in a single analyte peak [10] and, in another case, of as little as 100 DNA molecules/band [11]. Tagging DNAs with flourophores which emit in the near infrared $(830$ $\mathrm{nm}$ ) has further improved the signal by at least one order of magnitude as compared with conventional fluorescent dyes emitting at around 500 to $550 \mathrm{~nm}$ [12]. Optimization of the buffer components has dramatically improved the performance of CZE in 
DNA analysis. Thus, Gelfi et al. [13] have recently shown that the use of isoelectric buffers (notably 100 $\mathrm{m} M$ His) at their $\mathrm{p} I$ values $(\mathrm{pH}=\mathrm{p} I=7.47$ for His) as the sole buffering ion dramatically shortens the separation times, while maintaining excellent resolution.

Yet, notwithstanding all these major innovations, one field which has not experienced any dramatic progress is the chemistry of the sieving liquid polymers, typically dissolved in the background electrolyte for implementing size separation of DNA fragments. An interesting recent development has been the synthesis of the novel monomer, $\mathrm{N}$-acryloyl amino ethoxy ethanol (AAEE), combining high hydrophilicity with high stability in an alkaline milieu [14]. Poly(AAEE), when used in DNA separations, provided a better performance than conventional poly(acrylamide) [15]. Yet, as recently reported by us $[16,17]$, AAEE could not be prepared as a polymer of reduced and controlled length, since under conditions leading to the growth of polymers of shorter chain-length, it auto-reticulated, forming a solid gel, by the mechanism of "1-6 abstraction". On the other hand, poly(acrylamides) of reduced chain length are more desirable in DNA separations because: (a) they have much reduced viscosities, so that they can be easily extruded and injected in a capillary; (b) offer very good resolution and plate counts in a DNA size window between 100 and 1000 bp, i.e., the most informative DNA size range in molecular biology, genetic analysis, forensic medicine and most life science disciplines [18]. As an additional drawback, AAEE solutions, upon prolonged storage, were much more prone to autopolymerize than standard acrylamide or other $\mathrm{N}$ substituted acrylamides. In view of these shortcomings, we have synthesized [16] and characterized [17] a series of novel monomers, all bearing an $\Omega$-hydroxy substituent on the amido nitrogen, but devoid of the ether group present in the ethoxy moiety of AAEE and thought to be responsible for its noxious habit of auto-polymerizing and autocross-linking.

We report in the present paper the use of a series of $\Omega$-hydroxy, $\mathrm{N}$-substituted acrylamides (bearing, as $\mathrm{N}$-substituents, ethanol, or propanol, or butanol or pentanol moieties) as sieving media for analysis of DNA fragments and we compare their performances among themselves and vs. the conventional poly(acrylamide) viscous polymer solutions. Additionally, we report the properties of their co-polymers.

\section{Experimental}

\subsection{Reagents}

Acrylamide, Tris (hydroxymethylaminomethane), ammonium peroxodisulphate and $\mathrm{N}, \mathrm{N}, \mathrm{N}^{\prime}, \mathrm{N}^{\prime}$-tetramethylethylene diamine (TEMED) were obtained from Bio-Rad Labs. (Richmond, CA, USA). 3-(trimethoxysilyl) propylmethacrylate (Bind Silane) and poly(ethylene oxide) were from Aldrich (Steinheim, Germany). NAT (N-acryloyl-Tris or Trisacryl) was a kind gift from Dr. E. Boschetti, Rhone Poulenc, Villeneuve La Garenne, France. The series of $\mathrm{N}$ substituted acrylamide homologues (AAE: Nacryloyl amino ethanol; AAP: $\mathrm{N}$-acryloyl amino propanol; AAB: $\mathrm{N}$-acryloyl amino butanol; AAPen: $\mathrm{N}$-acryloyl amino pentanol) were synthesized and characterized in our lab according to Simò-Alfonso et al. [16,17]. N-acryloyl amino ethoxy ethanol (AAEE) was synthesized according to Chiari et al. [14]. Dimethyl acrylamide (DMA) was purchased from Sigma (St. Louis, MO, USA). Ethylendiamine tetraacetic acid (EDTA), boric acid and acetic acid were from Merck (Darmstadt, Germany). fused-silica capillaries (100 and $75 \mu \mathrm{m}$ I.D., $375 \mu \mathrm{m}$ O.D.) were obtained from Polymicro Technologies (Phoenix, AZ, USA). The DNA marker pBR322/ HaeIII was from Boehringer (Mannheim, Germany) whereas the 10-100 and 10-100 bp ladders were from Life Technologies (Gaithersburg, MD, USA).

\subsection{Capillary electrophoresis}

Capillary zone electrophoresis (CZE) was carried out with the Waters Quanta $4000 \mathrm{E}$ apparatus from Millipore (Milford, MA, USA); $37 \mathrm{~cm}$ long, 100 and 75 and $\mu \mathrm{m}$ I.D. capillaries, coated by a slight modification of Hjerten's protocol, utilizing $\mathrm{N}$ acryloyl amino ethoxy ethanol (AAEE) as monomer [14], filled with different viscous polymer solutions, were used. The sample and standard were loaded electrophoretically by applying $100 \mathrm{~V} / \mathrm{cm}$ for $6 \mathrm{~s}$. Separations were performed at $100 \mathrm{~V} / \mathrm{cm}$. Ultraviolet 
absorbance was monitored at $254 \mathrm{~nm}$. pBR322/ HaeIII (marker V), covering an 8 to $587 \mathrm{bp}$ range, and the 10-100 bp ladder were used as markers for evaluating the separation performance.

\subsection{Viscosity measurements}

Viscosity measurements of polymer solutions were performed on a Bohlin VOR rheometer (Bohlin Rheology AB, Lund, Sweden) using the rotation method, with a cone/plate measuring system (CP5/ 30) (5.4 rad cone angle, $30 \mathrm{~mm}$ diameter), a gap of $0.15 \mathrm{~mm}$ and torsion bars of $4.13 \mathrm{~g} \cdot \mathrm{cm}$ and of 20.83 $\mathrm{g} \cdot \mathrm{cm}$. The sample amount was about $0.7 \mathrm{ml}$, enough to cover the plate surface. Viscosity was evaluated, at constant concentration, as a function of temperature in the $10-60^{\circ} \mathrm{C}$ interval, with a heating rate of $2^{\circ} \mathrm{C} / \mathrm{min}$ and a shear rate of $293.8 \mathrm{~s} \mathrm{~s}^{-1}$. Viscosity is expressed in $\mathrm{mPa} \cdot \mathrm{s}$, the viscosity of water at $25^{\circ} \mathrm{C}$ being 1 .

\section{Results}

Fig. 1 shows the separation of marker V DNA fragments in different types of polymers and copolymers obtained with $\Omega$-hydroxy $\mathrm{N}$-substituted acrylamides. While all separations seem to be of very high quality, some interesting trends can be appreciated. First of all, while the train of bands, in pure $6 \%$ poly(AAP) is eluted in a time window of 30 to $55 \mathrm{~min}$, spiking this polymer with increasing amounts of the butanol derivative (e.g., 4\% AAP copolymerized with $2 \% \mathrm{AAB}$ ) shifts the patterns to longer transit times. In a pure poly( $\mathrm{AAB}$ ) entangled solution, although more dilute (5\%), the elution window increases to 35 to $60 \mathrm{~min}$. These shifts in transit times are accompanied by a marked increase in theoretical plate numbers, as summarized in Table 1. It is seen that, whereas in a pure solution of $6 \%$ poly(AAP) the plate number (averaged over all peaks) is 880000 , in a mixed co-polymer AAP$\mathrm{AAB}(5: 1)$ it increases to 946000 and in the same $\mathrm{AAP}-\mathrm{AAB}$ co-polymer at a $4: 2$ ratio it reaches a level of 1050000 . In a pure poly $(\mathrm{AAB})$ polymer (at only $5 \%$ level) the plate count is as high as 1200000 . Table 1 also summarizes other data not presented in Fig. 1. Thus, it is seen that the shortest

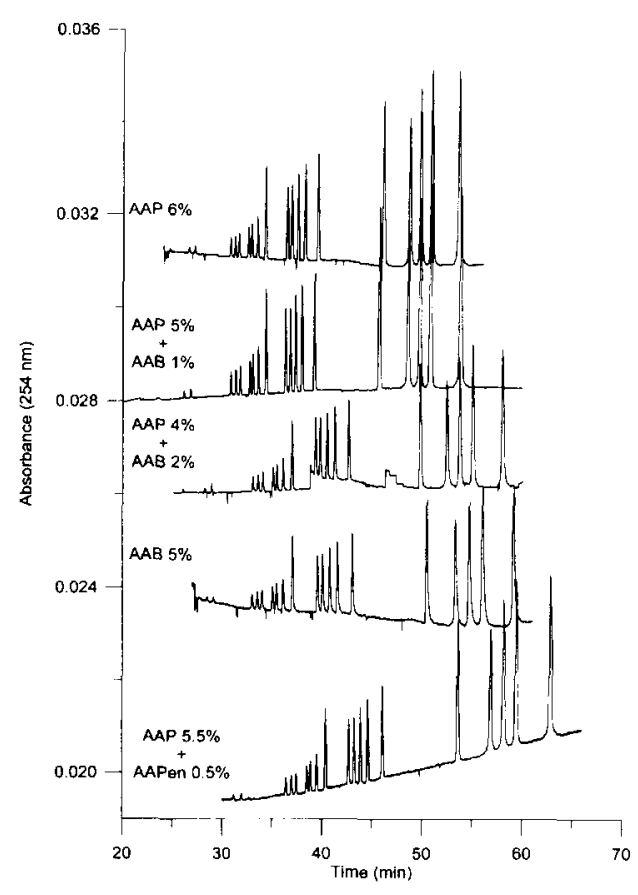

Fig. 1. Separation of marker V DNA fragments by CZE in entangled polymer solutions. Polymers (from top to bottom): $6 \%$ poly(AAP); co-polymer formed by $5 \%$ AAP mixed with $1 \%$ AAB; co-polymer formed by $5 \%$ APP mixed with $2 \%$ AAB; $5 \%$ poly $(\mathrm{AAB})$ and copolymer of $5.5 \% \mathrm{AAP}$ with $0.5 \%$ AAPen. Background electrolyte: TBE buffer, pH 8.3 . Capillary: $37 \mathrm{~cm}$ long, $100 \mu \mathrm{m}$ I.D. The sample was loaded electrophoretically by applying $100 \mathrm{~V} / \mathrm{cm}$ for $6 \mathrm{~s}$. Separations were performed at 100 $\mathrm{V} / \mathrm{cm}$. Ultraviolet absorbance was monitored at $254 \mathrm{~nm}$. The ds-DNA fragments cover the 8 to 587 bp size range.

$\mathrm{N}$-substituted acrylamide (the ethanol derivative, $\mathrm{AAE}$ ) does not have much to offer in terms of resolution: in an $8 \%$ solution of poly(AAE) (and in narrower bore capillary, of $75 \mu \mathrm{m}$ ) the plate count is only 670000 , increasing to 850000 in a $10 \%$ viscous polymer solution. The longest monomer of the series (the pentanol derivative, AAPen), although not very useful in DNA separations as a pure polymer (possibly due to its hydrophobicity), gave very good results when spiked at low levels in the propanol derivative: a mixture of 5.5:0.5 AAP/ AAPen produced a plate number of 1100000 . As shown in Table 1, a series of runs at different concentrations of poly(AAP) gave progressively higher plate counts, which peaked at $6 \%$ polymer and then slowly declined.

Fig. 2 shows the separation of the $10-100 \mathrm{bp}$ 
Table 1

performance of different $\mathrm{N}$-substituted acrylamides (as pure polymers or co-polymers) in DNA separations

\begin{tabular}{lllcr}
\hline Monomer & Concentration $(\%)$ & Buffer & I.D. $(\mu \mathrm{m})$ & Theoretical plates \\
\hline AAE & 8 & TBE & 75 & 670000 \\
& 10 & TBE & 75 & 850000 \\
AAP & 1.5 & TBE & 75 & 467000 \\
& 3 & TBE & 75 & 922000 \\
& 6 & TBE & 75 & 820000 \\
& 8 & TBE & 75 & 880000 \\
AAP+AAB & 6 & TBE & 100 & 710000 \\
AAP+AAB & 6 & Tris/Acet & 100 & 946000 \\
AAP+AAPen & $5: 1$ & TBE & 100 & 1050000 \\
AAB & $4: 2$ & TBE & 100 & 1100000 \\
AAEE & $5.5: 0.5$ & TBE & 100 & 1200000 \\
\hline
\end{tabular}

ladder in pure $5 \%$ poly( $\mathrm{AAB})$. The excellent resolution obtained can be observed in the fact that the early eluting fragments tend to be split into a doublet, possibly due to trace side-reaction occurring during the synthesis.

Fig. 3 gives a $\log -\log$ plot of mobility vs. molecular size (in $M_{\mathrm{r}}$ ) for all the fragments run in Fig. 1, in the different polymer/copolymer solutions. According to Noolandi [19], this plot should evidence three regimes: the first linear portion to the left

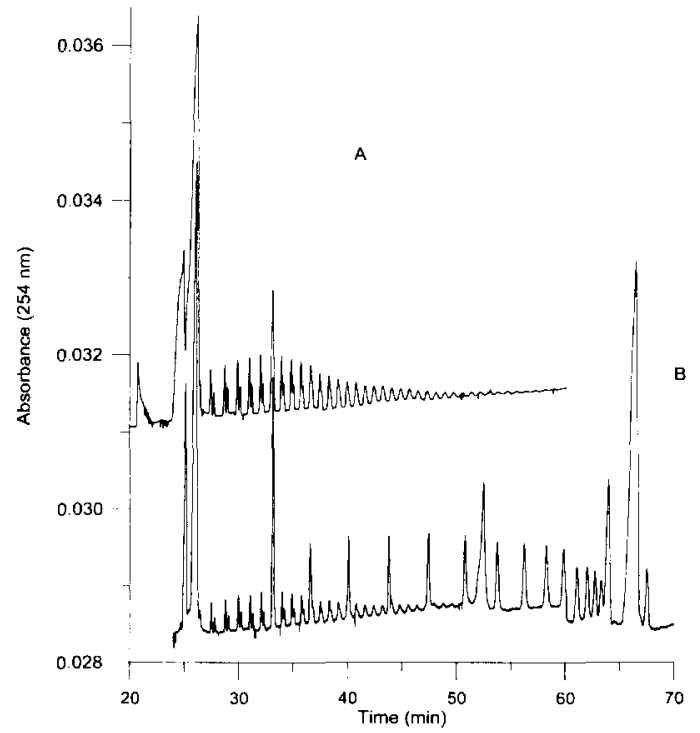

Fig. 2. Separation of the $10+100 \mathrm{bp}$ (upper curve, A) and of the $100-1000$ bp (lower tracing, B) DNA ladders by CZE in $5 \%$ poly (AAB) in TBE buffer, $\mathrm{pH}$ 8.3. All other conditions as in Fig. 1. being DNA migration according to the Ogston sieving model, the middle portion DNA movement according to a reptation-without stretching regime and the last portion (absent in our Figure due to the relatively small size of our fragments), being a reptation-with-stretching regime, at which size resolution is lost and all DNA fragments migrate with the same mobility. This theoretical plot was first experimentally verified by us in capillary electro-

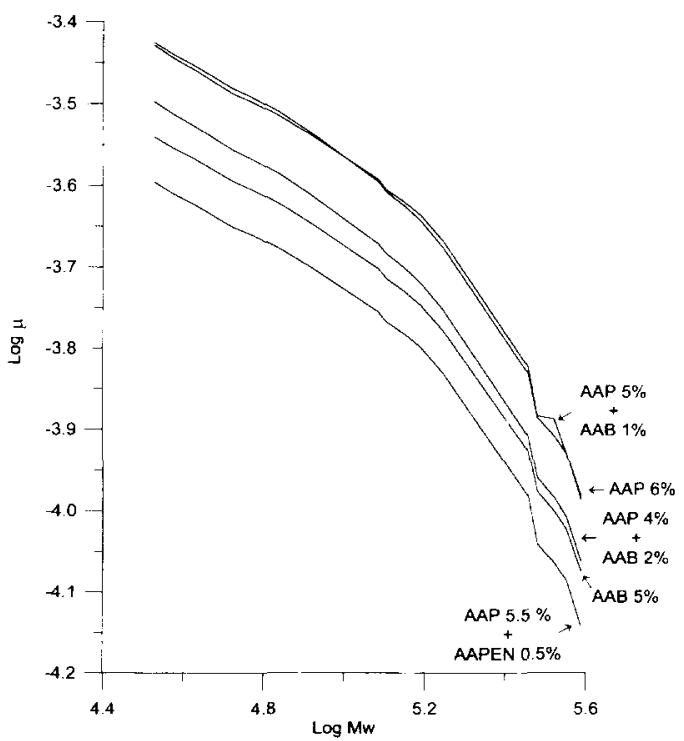

Fig. 3. Double $\log$ plot of mobility vs. size (in $M_{\mathrm{r}}$ ) of the DNA fragments separated in Fig. 1, in presence of the different sieving polymers and co-polymers. Note that the reptation-withoutstretching regime begins around a fragment size of $300 \mathrm{bp}$. 
phoresis [20] and independently by Magnusdottir et al. [21] by linear dicroism studies. As shown in Fig. 3 , the series of different polymers/copolymers gives a family of curves which are parallel in the first part (migration according to Ogston) and then tend to converge to some extent in the right portion. Interestingly, the regime of reptation-without-stretching tends to manifest itself for relatively small DNA particles, of the order of $300 \mathrm{bp}$, whereas, according to Noolandi [22], this migration regime should appear $>1000 \mathrm{bp}$.

We were intrigued by both the marked increase in plate counts in the polymers/copolymers shown in Table 1 and the early onset of the reptation regime in these viscous polymer solutions. In order to understand these phenomena, we have resorted to viscosity measurements as a function of both increasing temperatures and buffer composition of the polymer mixtures. Fig. 4 gives the results of these experiments: as shown in the bottom tracing, $5 \%$ poly(AAP), when dissolved in TBE buffer, shows a viscosity profile slowly declining with temperature till plateauing at around $50^{\circ} \mathrm{C}$. A similar behaviour is exhibited by the same poly(AAP) when dissolved in

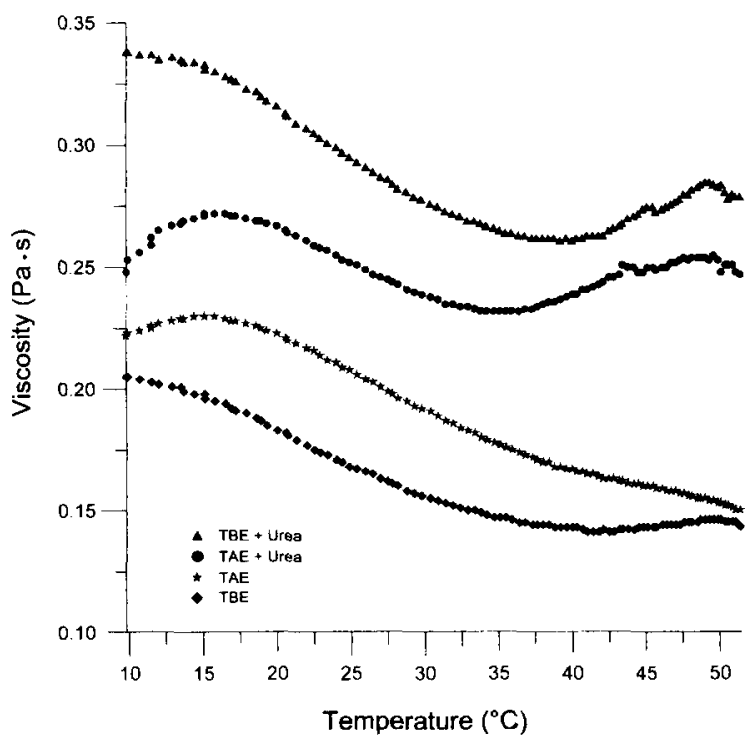

Fig. 4. Viscosity vs. temperature plots of poly(AAP) dissolved in: $89 \mathrm{~m} M$ TBE buffer, $\mathrm{pH} 8.3$ (lower tracing, diamonds); in $89 \mathrm{mM}$ TBE buffer, $\mathrm{pH} 8.3$, in presence of $6 M$ urea (upper tracing, triangles); in $89 \mathrm{mM}$ TAE buffer, $\mathrm{pH} 8.3$ (stars) and in $89 \mathrm{mM}$ TAE buffer, pH 8.3, added with $6 M$ urea (solid circles).
TAE (Tris-acetate-EDTA) buffer. Curiously, however, the viscosity in this latter buffer has a quite different profile: it is in all cases higher than in TBE buffer, peaks at around $18^{\circ} \mathrm{C}$ and then it declines to almost coincident values at $50^{\circ} \mathrm{C}$ as in TBE. Surprisingly, the viscosity of $5 \%$ poly(AAP) in TBE almost doubles in presence of $6 M$ urea, it reaches a minimum at $40^{\circ} \mathrm{C}$ to increase slightly and peak at $50^{\circ} \mathrm{C}$. The curve in TAE in presence of $6 \mathrm{M}$ urea follows, in the first half of the profile, the one in absence of urea: it peaks at $18^{\circ} \mathrm{C}$ too. However, whereas in plain TAE the curve continuously declines, here it reaches a minimum at $37^{\circ} \mathrm{C}$ and then climbs up again, in a profile very similar now to the one presented in TBE/urea.

The reason for the different behaviour and shape of these curves is presently not understood, but at least it dispels a working hypothesis we had made when exploring the behaviour of the different $\Omega$ hydroxy, $\mathrm{N}$-substituted acrylamides: i.e., the possibility of forming borate-diol complexes, with borate acting as a "cross-linker" between different polymer chains. This might have explained the steady increase in plate number when using our family of $\Omega$-hydroxy acrylamides. But this hypothesis is clearly not tenable, since, in the case of borate acting as "cross-linker", the viscosity increment would have been enormous [23,24], whereas in our case the viscosity is even higher in plain TAE buffer. Also the behaviour of these polymers in presence of urea is quite puzzling: given the fact that urea is a typical H-bond breaker, and that a plain $6 M$ urea solution has a rather low viscosity, the marked viscosity increments in both buffer systems, coupled to the odd shape of the curves, remains at present unexplained.

\section{Discussion}

In recent years, there has been a renewed interest in exploring the use of $\mathrm{N}$-substituted acrylamides as novel sieving matrices. Already in 1987-1988 Kozulic's group $[25,26]$ proposed the use of NAT as a novel anticonvective matrix for protein and DNA separations. Curiously, at the same time, our group also explored the use of poly(NAT) [or poly(Trisacryl)] for isoelectric focusing, but our enthusiasm 
on this uniquely hydrophilic matrix was substantially quenched when we discovered that the gel was disintegrating under the cathodic filter paper strip [27]. While on the one side Kozulic and Mosbach patented their poly(NAT) matrix (US Patent $5319046,1994)$ we started looking for different monomers after discovering that NAT had the nasty habit of degrading with zero-order kinetics [28]. Just about at the same time, Jain and Shorr proposed Hydrolink matrices, which however turned out to be mainly composed of dimethyl acrylamide, a highly stable, but highly hydrophobic monomer [29]. The above findings led us to design the $\mathrm{N}$-acryloyl amino ethoxy ethanol monomer, combining high hydrophilicity with extreme hydrolytic stability [14]. As it turns out now, even this last monomer, although leaving up to our expectations and performing very well in DNA separations [15], was born with a hidden "genetic defect" which took long time to be discovered: its pronounced tendency to auto-polymerize and auto-reticulate (in the absence of a crosslinker!) by a unique mechanism of " 1,6 abstraction". As this instability was traced back to the presence of an ether oxygen (in the ethoxy moiety) in the N-substituent chain, the novel class of monomers was designed [16,17], based on plain alcohol derivatives in a homologous series (from ethanol to pentanol). As shown in Table 1, and in the various figures, it would appear that the best monomers, in terms of efficiency of DNA separations, are the $\mathrm{N}$-propanol (AAP) and N-butanol (AAB) substituents. Below and above the length of the $\mathrm{N}$ substituent chains of these two compounds, DNA separations rapidly deteriorate. The N-ethanol substituent (AAE) gave us poor resolutions, possibly also due to the same fast degradation process typical of NAT, induced by the mechanism of "N-O acyl transfer" [28]. Separations rapidly worsened also with the $\mathrm{N}$-pentanol substituent (AAPen). However, in this last case this is possibly due to the relatively high hydrophobicity of the pentanol chain (the partition coefficient $P$ of this last monomer, 0.5 , is essentially the same as that of dimethyl acrylamide). This, however, leaves us with two outstanding monomers to play with in DNA separations: AAP and $\mathrm{AAB}$ (and their co-polymers), both possessing extreme resistance to hydrolysis, both being quite hydrophilic ( $P$ values: 0.1 for AAP, 0.2 for acrylamide and 0.24 for $A A B$ ) and both being, of course, immune from the auto-reticulation and auto-polymerization pathways of $\mathrm{N}$-acryloyl amino ethoxy ethanol, their precursor in the chain of synthetic events reported above.

\section{Note added in proof}

After this manuscript had been accepted, we became aware of a paper [30] describing a novel method for calculating $N$, which takes into account peak assymmetry. In our case, we have used the standard way of calculating $N$, as incorporated in the Millenium software and, we believe, in most programs handling CZE data. The standard procedure assumes Gaussian peaks and calculates $N$ on the basis of peak width at half height. Thus we cannot exclude the possibility that our data could be biased in case of a strong peak assymmetry.

\section{Notation}

$\begin{array}{ll}\text { AAEE } & \mathrm{N} \text {-acrylyol amino ethoxy ethanol } \\ \text { DMA } & \mathrm{N} \text {-dimethyl acrylamide } \\ \text { AAP } & \mathrm{N} \text {-acryloyl amino propanol } \\ \text { NAT } & \text { Trisacryl } \\ \text { AAB } & \mathrm{N} \text {-acryloyl amino butanol } \\ \text { AAPen } & \mathrm{N} \text {-acryloyl amino pentanol } \\ \text { AAE } & \mathrm{N} \text {-acryloyl amino ethanol }\end{array}$

\section{Acknowledgments}

P.G.R. is supported by a grant from the European Community (Bio Med 2, Human Genome Sequencing, No. BMH4-CT96-1158), by Consiglio Nazionale delle Ricerche (CNR, Roma, Progetto Strategico 95.04567.ST74) and by Telethon Italy (grant No. E-153). E.S.A. thanks the European Community for financial support (Human Capital and Mobility Programme, bursary No. BMHI-CT947518).

\section{References}

[1] P.G. Righetti and C. Gelfi, in P.G. Righetti (Editor), Capillary Electrophoresis: an Analytical Tool in Biotechnology, CRC Press, Boca Raton, FL, 1996, pp. 431-476. 
[2] R.A. Mathies and X.C. Huang, Nature, 359 (1992) 167-169.

[3] X.C. Huang, M.A. Quesada and R.A., Mathies, Anal. Chem., 64 (1992) 967-972.

[4] X.C. Huang, M.A. Quesada and R.A. Mathies, Anal. Chem., 64 (1992) 2149-2154.

[5] S.M. Clark, R.A. Mathies, Anal. Biochem., 215 (1993) $163-170$.

[6] Y. Wang, J. Ju, B.A. Carpenter, J.M. Atherton, G.F Sesabaugh and R.A. Mathies, R.A., Anal. Chem., 67 (1995) 1197-1203.

[7] A. Manz, D.J. Harrison, E.M.J. Verpoorte, C.J. Fettinger, A. Paulus, H. Ludi and H.M. Widmer, J. Chromatogr., 593 (1992) 253-258

[8] A.T. Woolley and R.A. Mathies, Proc. Natl. Acad. Sci. USA, 91 (1994) $11348-11352$.

[9] A.T. Woolley and R.A. Mathies, Anal. Chem., 67 (1995) 3676-3680

[10] A. Castro and E.B. Shera, Anal. Chem., 67 (1995) 31813186.

[11] B.B. Haab and R.A. Mathies, Anal. Chem., 67 (1995) $3253-3260$

[12] D.C. Williams and S.A. Soper, Anal. Chem.. (67) 1995 3427-3432.

[13] C. Gelfi, M. Perego and P.G. Righetti, Electrophoresis, 17 (1996) in press.

[14] M. Chiari, C. Micheletti, M. Nesi, M. Fazio and P.G. Righetti, Electrophoresis, 15 (1994) 177-186.

[15] M. Chiari, M. Nesi and P.G. Righetti, Electrophoresis, 15 (1994) 616-622.
[16] E. Simò-Alfonso, C. Gelfi, R. Sebastiano, A. Citterio and P.G. Righetti, Electrophoresis, 17 (1996) 723-731.

[17] E. Simò-Alfonso, C. Gelfi, R. Sebastiano, A. Citterio and P.G. Righetti, Electrophoresis, 17 (1996) 732-737.

[18] C. Gelfi, A. Orsi, F. Leoncini and P.G. Righetti, J. Chromatogr. A, 689 (1995) 97-105

[19] J. Noolandi, Advan. Electrophoresis, 5 (1992) 1-57.

[20] M. Chiari, M. Nesi and P.G. Righetti, J. Chromatogr. A, 652 (1993) 31-39

[21] S. Magnusdottir, B. Akerman and M. Jonsson, J. Phys. Chem., 98 (1994) 2624-2633.

[22] C. Turmel, E. Brassard and J. Noolandi, Electrophoresis, 13 (1992) 620-622.

[23] P.G. Righetti and R.S. Snyder, Theor. Applied Electr., 1 (1988) $53-58$

[24] P.D. Grossman and D.S. Soane, J. Chromatogr., 559 (1991) 257-265

[25] M. Kozulic, B. Kozulic and K. Mosbach, Anal. Biochem., 163 (1987) $506-512$.

[26] B. Kozulic, K. Mosbach and M. Pietrzak, Anal. Biochem., 170 (1988) 478-484.

[27] P. G. Righetti, C. Gelfi, M.L. Bossi and E. Boschetti, Electrophoresis, 8 (1987) 62-70.

[28] C. Gelfi, P. De Besi, A. Alloni and P.G. Righetti, J. Chromatogr., 608 (1992) 333-341.

[29] R.J. Molinari, M. Connors and R.G.L. Shorr, Adv. Electrophoresis. 6 (1994) 43-60.

[30] M.S. Jeansonne and J.P. Foley, J. Chromatogr. Sci., 29 (1991) 258-266. 